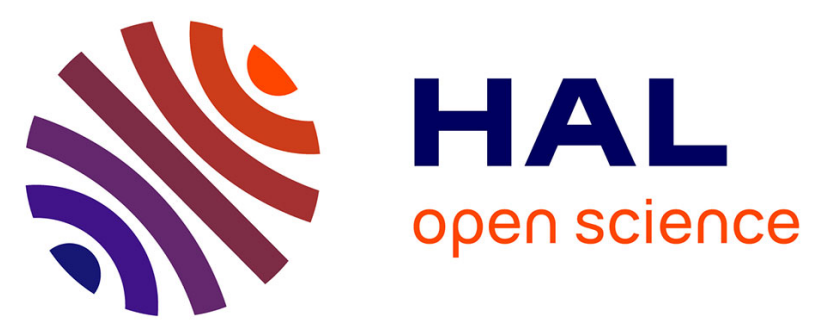

\title{
Observation-Based Nonlinear Proportional-Derivative Control for Robust Trajectory Tracking for Autonomous Underwater Vehicles
}

Jesus Guerrero, Jorge Torres, Vincent Creuze, Ahmed Chemori

\section{- To cite this version:}

Jesus Guerrero, Jorge Torres, Vincent Creuze, Ahmed Chemori. Observation-Based Nonlinear Proportional-Derivative Control for Robust Trajectory Tracking for Autonomous Underwater Vehicles. IEEE Journal of Oceanic Engineering, 2020, 45 (4), pp.1190-1202. 10.1109/JOE.2019.2924561 . lirmm-02281181

\section{HAL Id: lirmm-02281181 \\ https://hal-lirmm.ccsd.cnrs.fr/lirmm-02281181}

Submitted on 9 Sep 2019

HAL is a multi-disciplinary open access archive for the deposit and dissemination of scientific research documents, whether they are published or not. The documents may come from teaching and research institutions in France or abroad, or from public or private research centers.
L'archive ouverte pluridisciplinaire HAL, est destinée au dépôt et à la diffusion de documents scientifiques de niveau recherche, publiés ou non, émanant des établissements d'enseignement et de recherche français ou étrangers, des laboratoires publics ou privés. 


\title{
Observation Based Nonlinear PD Control For Robust Trajectory Tracking For Autonomous Underwater Vehicles
}

\author{
J. Guerrero, J. Torres, V. Creuze, and A. Chemori
}

\begin{abstract}
This paper deals with the design, improvement, and implementation of a nonlinear control strategy to solve the trajectory tracking problem for an Autonomous Underwater Vehicle (AUV) under model uncertainties and external disturbances. First, a disturbance observer based on High Order Sliding Mode Control is designed in order to counteract the negative impact of both parametric uncertainties and bounded external disturbances. Then, the nonlinear control is enhanced through injecting the disturbance estimation into the designed controller. The stability of the closed-loop system with the enhanced proposed nonlinear controller is proven by Lyapunov arguments. Finally, real-time experimental results are also provided to demonstrate the effectiveness of the proposed controller.
\end{abstract}

\section{Index Terms}

Extended State Observer, Proportional Derivative, High Order Sliding Mode Control, Underwater Vehicles, trajectory tracking control, Disturbance observer.

\section{INTRODUCTION} operated underwater vehicles (ROVs), which require human piloting and, on the other hand,

The Leonard underwater vehicle has been financed by the European Union (FEDER grant $\mathrm{n}^{\circ}$ 49793) and the Region Occitanie (ARPE Pilot Plus project).

J. Guerrero and J. Torres are with the Center for Research and Advanced Studies of the National Polytechnic Institute (CINVESTAV), Mexico City, MX, 07360, Mexico (e-mail: jguerrero@ctrl.cinvestav.mx;jtorres@ctrl.cinvestav.mx).

V. Creuze and A. Chemori are with the Montpellier Laboratory of Computer Science, Robotics, and Microelectronics (LIRMM) of the University of Montpellier, 161 rue Ada 34095 Montpellier Cedex 5 - France (e-mail: vincent.creuze@lirmm.fr; ahmed.chemori@lirmm.fr). 
the Autonomous Underwater Vehicles (AUVs), that refer to the submarines able to perform some tasks with full autonomy. In recent years, the scientific community has been interested in expanding the autonomy condition offered by this class of vehicles.

There are several typical control tasks to provide autonomy to a submarine vehicle. Among them, one can cite: 1) Point stabilization which refers to the problem of steering a vehicle to a final target point. 2) Path following control which aims at forcing a vehicle to converge to and follow a desired spatial path. 3) Path tracking which makes a vehicle track a timeparameterized reference curve [1]. In this work, we focus on the latter case, where the design of an AUV path-tracking controller is not a trivial task due to its complex and highly nonlinear dynamics of the vehicle and the difficulty in accurately modeling the hydrodynamic effects. Moreover, unpredictable external perturbations (impacts, ocean currents,etc) are likely to happen, thus complicating the control task.

There is a wide range of control techniques applied to underwater vehicles: For example, Proportional-Derivative (PD) and Proportional Integral Derivative (PID) controls are the most used techniques to control the position and orientation of commercial AUVs due to their design simplicity and very good performances [2], [3], [4]. However, it is well-know that the PID control performance is degraded when the plant to be controlled is highly nonlinear, time varying, or with significant time delay. The impact of the mentioned drawbacks can be diminished by using nonlinear PD/PID schemes. For example, nonlinear PID controllers with the anti-windup design [5] or using nonlinear functions [6]. For instance, an AUV trajectory tracking control based on the Nonlinear PD (NLPD) strategy was proposed by [7]. In this work, the authors show the main advantages of the NLPD design over the classic PD control under several operating conditions. Based on the experimental results, the NLPD shows a good trajectory tracking behavior but its performance is degraded against persistent external disturbances and excessive parametric uncertainties as it can be seen through the depth trajectory tracking test results. In the mentioned test, there is a significant tracking error in the steady state due to the considerable buoyancy added to the submarine L2ROV. Finally, the authors suggested that the introduction of an integral term to the NLPD algorithm will minimize the tracking error.

On the other hand, a broad class of robust controllers have been proposed for the path tracking problem on AUV. For example, Fuzzy Logic Controllers (FLC) [8], Neural-Network based control (NNC) [9], Adaptive control [10], [11], [12], [13], Sliding Modes Control (SMC) [14], High Order Sliding Modes Control (HOSMC) [15], [16], [17] and so on. As expected, 
each methodology has strengths and weaknesses. For example, FLC have a simple structure, easy and cost-effective design. Nonetheless, the controller tuning process might be a bit difficult because there is no stability criterion or FLC cannot be implemented for unknown system of no information.

The fundamental advantage of NNC is their ability to learn from examples instead of requiring an algorithmic development from the designer. However, NNC usually needs a long and computationally expensive training time which is not acceptable in many applications.

Adaptive control covers a set of techniques which provide a systematic approach for automatic adjustment of controllers in real time, in order to achieve or to maintain a desired level of system performance when the parameters of the dynamic model are unknown and/or change in time [18]. For example, in [19] the so-called L1 adaptive control was applied for depth and pitch trajectory control for an AUV. From the experimental results, the authors showed the advantages of this kind of controller which can re-tune its control gains in spite of external disturbances. The main disadvantage of this control technique is the parameter estimation low rate.

Sliding Mode Control (SMC) is another robust technique sometimes used in underwater vehicle control. This technique provides finite time convergence and robustness against bounded external disturbances. In its basic implementations, this controller can have aggressive control input behavior due to signum function which causes the undesirable chattering effect. However, there exists several ways to decrease the chattering effect, like replacing the signum function by a hyperbolic tangent function [2], [20], or implementing High Order Sliding Mode Control (HOSMC) which takes advantage of quasi-continuous control [17], [21], or using controllers with dynamic gains, in these techniques, an adaptive law is proposed to adjust the controller gains. For instance, in [22] an adaptive Generalized Super-Twisting Algorithm (GSTA) for trajectory tracking for AUV is proposed. In [23], an adaptive second-order fast nonsingular terminal sliding mode control (ASFNTSMC) is proposed to solve the trajectory tracking control problem of fully actuated AUV under parametric uncertainties and external disturbances. In [24], two adaptive integral schemes, namely, Adaptive Integral Terminal SMC (AITSMC) and Adaptive Fast Integral Terminal SMC (AFITSMC) was proposed for the trajectory tracking control problem of Underwater vehicles under dynamic uncertainties and time-varying external disturbances.

In brief, nonlinear controllers show a wide range of advantages over non-robust techniques. However, these methods involve complex design. For this reason, controllers such as PD or PID 
are improved through their fusion with algorithms of estimation of parametric uncertainties and external disturbances. For instance, a SMC enhanced by uncertainty and disturbance estimator (UDE) for an AUV tracking control in steering and diving planes is shown in [25]. In this article, the discontinuous action of the SMC is replaced by the disturbance estimation made by the UDE algorithm offering a chatter-free controller. However, the algorithm is designed based on the AUV linearized system. Moreover, the proposed control law use the equivalent control method, which means that the full knowledge of the system is necessary. Then, the designed controller is compared with the classical PID and SMC through computer simulations. Finally, the authors show the superior performance of the proposed scheme over the listed methods.

On the other hand, a Backstepping (BS) control with exponential convergence improved by the mixture with a lumped uncertainty observer is shown in [26]. In this paper, the authors design a lumped uncertainty observer with a simple structure. Then, the estimated disturbance signal is injected into the BS controller to compensate the external disturbances. Finally, through computer simulations, the authors demonstrate the enhancement of the proposed methodology with respect to the original BS control design. However, the proposed controller has six control gains to tune, and there is not a precise method to tune the observer gains. Moreover, there is a substantial compromise between the disturbance estimation and the controller's convergence velocity rate.

With respect to the disturbance observation problem, the extended state observer (ESO) methodology is applied to an AUV trajectory tracking in [27]. In this work, the authors propose an adaptive ESO algorithm to estimate the unknown submarine velocity, parametric uncertainties and external disturbances for the full six degrees of freedom (DoF) system. Then, an integral sliding mode control (ISMC) is designed where the disturbance estimation made by the ESO is used on the control law. Based on real-time experiments, the authors show the improvement to the ISMC and compare the algorithm against the classical PD controller. Nonetheless, the proposed control scheme needs the adjustment of many controller gains which can be timeconsuming. Also, the control law uses the signum function which causes chattering as can be seen on the control input graphs. Finally, although the algorithm was designed to compensate parametric uncertainties, the authors do not show an experiment modifying the parameters of the vehicle.

In this paper, in order to improve the NLPD controller shown in [7], an ESO based on the Generalized Super-Twisting Algorithm [28] is proposed. The enhanced NLPD (eNLPD) 
controller is constructed by injecting the disturbance observer into the control law. The extended state observer based on GSTA (GSTA-ESO) estimates the parametric uncertainties and the bounded external disturbances as well. The main contributions of this paper are as follows:

1) The GSTA-ESO is developed to estimate and compensate the parametric uncertainties and bounded external disturbances of the AUV trajectory tracking control. Then, the stability analysis of the controller plus disturbance observer can be carried out employing Lyapunov's arguments as shown in this contribution.

2) The GSTA-ESO improves the NLPD controller's performance shown in [7].

3) Compared with references [25], [26], [27], the proposed observer methodology only has three gains to tune. Moreover, a simple algorithm to tune the observer gains is provided.

4) The effectiveness of the proposed eNLPD is demonstrated through real-time experiments.

The rest of the paper is organized as follows: a brief description of the dynamic model of the submarine is given in Section 2. The enhanced proposed control technique is described in Section 3. The real-time experimental results for two DoF trajectory tracking are presented and analyzed in Section 4. Finally, some concluding remarks, ongoing and future work about the proposed controller are delineated in Section 5.

\section{Dynamic Model}

The dynamic model of underwater vehicles has been described in several references as in [2], [29], [30], [31].

The dynamics of an underwater vehicle involves two frames of reference: the body-fixed frame and the earth-fixed frame (as illustrated in Figure 11). Considering the generalized inertial forces, the hydrodynamic effects, the gravity, and buoyancy contributions as well as the effects of the actuators (i.e. thrusters), the dynamic model of an underwater vehicle in matrix form, using the SNAME notation [32] and the representation described in [2], can be written as follows:

$$
\begin{aligned}
M \dot{\nu}+C(\nu) \nu+D(\nu) \nu+g(\eta) & =\tau+w_{e} \\
\dot{\eta} & =J(\eta) \nu
\end{aligned}
$$

Where $\nu=[u, v, w, p, q, r]^{T}$ is the vector of velocity in the body-fixed frame and $\eta=[x, y, z, \phi, \theta, \psi]^{T}$ represents the vector of position and orientation in the earth-fixed frame. From equation (1) the matrix of spatial transformation between the inertial frame and the frame of the rigid body can be defined through the transformation of the Euler angles $J(\eta) \in \mathbb{R}^{6 \times 6} . M \in \mathbb{R}^{6 \times 6}$ is the matrix 


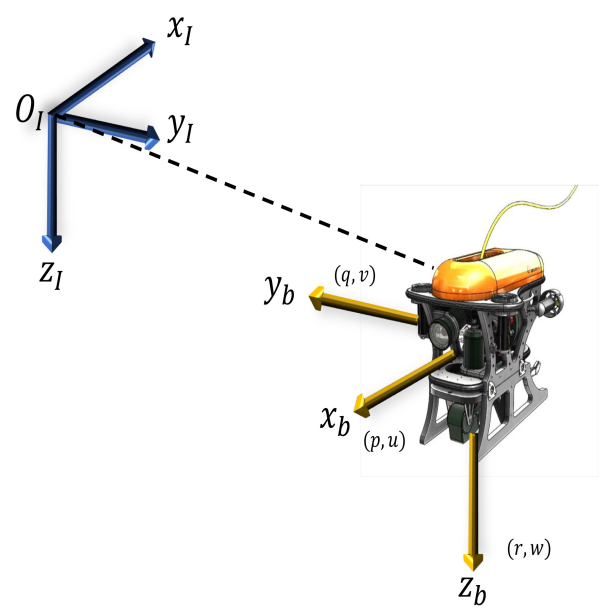

Fig. 1. Underwater vehicle reference frames. The inertial fixed on earth-fixed frame is denoted $\left(O_{I}, x_{I}, y_{I}, z_{I}\right)$ and the body fixed frame is denoted $\left(O_{b}, x_{b}, y_{b}, z_{b}\right)$.

of inertia where the effects of added mass are considered, $C(\nu) \in \mathbb{R}^{6 \times 6}$ is the Coriolis-centripetal matrix, $D(\nu) \in \mathbb{R}^{6 \times 6}$ represents the hydrodynamic damping matrix, $g(\eta) \in \mathbb{R}^{6}$ is the vector of gravitational/buoyancy forces and moments. Finally, $\tau \in \mathbb{R}^{6}$ is the control vector acting on the underwater vehicle, and $w_{e} \in \mathbb{R}^{6}$ represents the vector of external disturbances.

The dynamics (1) can be rewritten in the earth-fixed frame as (see [17] for more details):

$$
\underbrace{M_{\eta}(\eta) \ddot{\eta}+C_{\eta}(\nu, \eta) \dot{\eta}+D_{\eta}(\nu, \eta) \dot{\eta}+g_{\eta}(\eta)}_{f(\eta, \nu)}=\tau_{\eta}+w_{\eta}(t)
$$

It is difficult to accurately measure or estimate the hydrodynamic parameters [33]. As such, the system dynamics is roughly known. Therefore, the system dynamics $f(\eta, \nu)$ given in (3) can be written as the sum of estimated dynamics $\hat{f}(\eta, \nu)$ and the unknown dynamics $\tilde{f}(\eta, \nu)$ as follows:

$$
f(\eta, \nu)=\hat{f}(\eta, \nu)+\tilde{f}(\eta, \nu)
$$

where:

$$
\begin{aligned}
& \hat{f}(\eta, \nu)=\hat{M}_{\eta}(\eta) \ddot{\eta}+\hat{C}_{\eta}(\nu, \eta) \dot{\eta}+\hat{D}_{\eta}(\nu, \eta) \dot{\eta}+\hat{g}_{\eta}(\eta) \\
& \tilde{f}(\eta, \nu)=\tilde{M}_{\eta}(\eta) \ddot{\eta}+\tilde{C}_{\eta}(\nu, \eta) \dot{\eta}+\tilde{D}_{\eta}(\nu, \eta) \dot{\eta}+\tilde{g}_{\eta}(\eta)
\end{aligned}
$$


Moreover, the matrices of the unknown dynamics vector $\tilde{f}(\eta, \nu)$ are defined as $\tilde{M}_{\eta}=M_{\eta}-\hat{M}_{\eta}$, $\tilde{C}_{\eta}=C_{\eta}-\hat{C}_{\eta}, \tilde{D}_{\eta}=D_{\eta}-\hat{D}_{\eta}$ and $\tilde{g}_{\eta}=g_{\eta}-\hat{g}_{\eta}$.

Rewriting the system (3) into the estimated and unknown dynamics given by (4), we have:

$$
\hat{M}_{\eta}(\eta) \ddot{\eta}+\hat{C}_{\eta}(\nu, \eta) \dot{\eta}+\hat{D}_{\eta}(\nu, \eta) \dot{\eta}+\hat{g}_{\eta}(\eta)=\tau_{\eta}+\bar{d}(t)
$$

where the lumped unknown disturbance vector is defined as $\bar{d}(t)=w_{\eta}(t)-\tilde{f}(\eta, \nu)$.

\section{Disturbance Observer And Trajectory Tracking Controller Design}

A nonlinear PD controller for AUV trajectory tracking is developed in work [7]. In the cited paper, the authors propose a robust controller whose gains are nonlinear functions instead of the usual saturation function or constant gains for the PD controller as seen, for instance, in [6]. Based on the results of the real-time experiments, the authors prove the effectiveness and robustness of the proposed controller towards parametric uncertainties. However, the NLPD control is not able to reject the buoyancy disturbance on the submarine depth tracking test. In this paper, to counteract the mentioned controller deficiency, the performance of the NLPD controller is improved through the coupling of the disturbance observer. The disturbance observer scheme is based on the concept of total perturbation estimation via the GSTA [34]. The observer algorithm estimates the lumped unknown disturbance vector $\bar{d}(t)$ described in the model (7), and then, this estimation is injected to the controller to minimize the external disturbances impact on the performance of the AUV trajectory tracking task.

\section{A. Generalized Super-Twisting Algorithm Extended State Observer Design}

The ESO method was introduced initially by [35], [36]. This methodology is suitable only for integral chain systems. The main idea is to use an augmented state space model of the original system taking the disturbance term as an additional state. Then, a state observer is formulated for the new augmented system which will provide both the estimation of the system states as well as the matched disturbance. In this work, based on the ESO methodology described above and taking as reference the work presented in [37] we designed a new GSTA-ESO. From the AUV dynamics described by (7), we introduce the following state variables:

$$
\begin{aligned}
& \chi_{1}(t)=\eta(t) \\
& \chi_{2}(t)=\dot{\eta}(t)
\end{aligned}
$$


Rewriting the model (7) as follows:

$$
\begin{aligned}
& \dot{\chi}_{1}(t)=\chi_{2}(t) \\
& \dot{\chi}_{2}(t)=\bar{F}(\chi)+G(\chi) \tau_{\eta}+d(t)
\end{aligned}
$$

where:

$$
\begin{aligned}
\bar{F}(\chi) & =-\hat{M}_{n}(\eta)^{-1}\left[\hat{C}_{n}(\nu, \eta) \dot{\eta}+\hat{D}_{\eta}(\nu, \eta) \dot{\eta}+\hat{g}_{\eta}(\eta)\right] \\
G(\chi) & =\hat{M}_{n}(\eta)^{-1} \\
d(t) & =\hat{M}_{\eta}(\eta)^{-1} \bar{d}(t)
\end{aligned}
$$

To be able to construct the disturbance observer and the control law for the robot, it is necessary to introduce the following assumptions:

Assumption 1. The pitch angle is smaller than $\pi / 2$, i.e., $|\theta|<\pi / 2$.

Assumption 2. The external disturbance $d(t)$ is a Lipschitz continuous signal.

Remark. Underwater vehicles are not likely to enter the neighborhood of $\theta= \pm \pi / 2$ due to the metacentric restoring forces [24].

According to Assumption 1, the matrix $J(\eta)$ is not singular, therefore, its inverse exists. Also, according to Assumption 2, the time derivative of the lumped external disturbance terms $d(t)$ exists almost everywhere and it is bounded:

$$
\left|\dot{d}_{i}(t)\right| \leq L_{i}, \quad i=\overline{1,6}
$$

In order to design the GSTA-ESO for estimating the bounded disturbance $d(t)$ in (9), the auxiliary variable $\sigma$ is introduced

$$
\sigma(t)=\chi_{2}(t)+\Lambda \chi_{1}(t)
$$

where $\sigma(t):=\left[\sigma_{1}, \sigma_{2}, \cdots, \sigma_{6}\right]^{T}$ and $\Lambda=\operatorname{diag}\left(\lambda_{1}, \lambda_{2}, \lambda_{3}, \lambda_{4}, \lambda_{5}, \lambda_{6}\right)$ is a diagonal positive definite matrix. It is worth to note that the term $\Lambda$ modify the convergence rate of $\chi_{2}(t)$ to the origin when $\sigma(t)=0$.

The time derivative of the auxiliary variable $\sigma(t)$ is given by:

$$
\dot{\sigma}(t)=F(\chi)+G(\chi) \tau_{\eta}+d(t)
$$


with $F(\chi)=\bar{F}(\chi)+\Lambda \dot{\chi}_{1}(t)$.

As mentioned above, for design purpose, the total disturbance $d(t)$ in $(12)$ is considered as an extended state $\xi(t)$ as shown below:

$$
\begin{aligned}
\dot{\sigma}(t) & =F(\chi)+G(\chi) \tau_{\eta}+\xi(t) \\
\dot{\xi}(t) & =h(t)
\end{aligned}
$$

where $h(t)$ is the time derivative of the total disturbance $d(t)$.

The disturbance observer for the system (13) is constructed as follows:

$$
\begin{aligned}
\tilde{\sigma}(t) & =\hat{\sigma}(t)-\sigma(t) \\
\tilde{\xi}(t) & =\hat{\xi}(t)-\xi(t) \\
\dot{\hat{\sigma}} & =F(\chi)+G(\chi) \tau_{\eta}-K_{1} \Phi_{1}(\tilde{\sigma})+\hat{\xi}(t) \\
\dot{\hat{\xi}} & =-K_{2} \Phi_{2}(\tilde{\sigma})
\end{aligned}
$$

where $\tilde{\sigma}(t)$ and $\tilde{\xi}(t)$ are the estimation error of the ESO and the estimation error of the disturbance $d(t)$ respectively. $\hat{\sigma}(t)$ and $\hat{\xi}(t)$ are the observer internal states. $\dot{\hat{\sigma}}(t)$ and $\dot{\hat{\xi}}(t)$ are the dynamics of the observer internal states and the vectors $\Phi_{1}(\tilde{\sigma})=\left[\phi_{11}, \phi_{12}, \cdots, \phi_{16}\right]^{T}$ and $\Phi_{2}(\tilde{\sigma})=\left[\phi_{21}, \phi_{22}, \cdots, \phi_{26}\right]^{T}$ and each element of the mentioned vectors is given by:

$$
\begin{aligned}
& \phi_{1 i}\left(\tilde{\sigma}_{i}\right)=\mu_{1 i}\left|\tilde{\sigma}_{i}\right|^{1 / 2} \operatorname{sgn}\left(\tilde{\sigma}_{i}\right)+\mu_{2 i} \tilde{\sigma}_{i} \\
& \phi_{2 i}\left(\tilde{\sigma}_{i}\right)=\frac{1}{2} \mu_{1 i}^{2} \operatorname{sgn}\left(\tilde{\sigma}_{i}\right)+\frac{3}{2} \mu_{1 i} \mu_{2 i}\left|\tilde{\sigma}_{i}\right|^{1 / 2} \operatorname{sgn}\left(\tilde{\sigma}_{i}\right)+\mu_{2 i}^{2} \tilde{\sigma}_{i}
\end{aligned}
$$

where $\mu_{1 i}, \mu_{2 i} \geq 0$ with $i=\overline{1,6}, K_{1}=\operatorname{diag}\left(k_{11}, k_{12}, \cdots, k_{16}\right)$ and $K_{2}=\operatorname{diag}\left(k_{21}, k_{22}, \cdots, k_{26}\right)$ are the observer gains which are definite positive matrices.

Theorem 1. Consider the perturbed augmented system (13). The proposed GSTA-ESO (14) ensures that the observer error dynamics converges to zero in finite time if the gains $K_{1}$ and $K_{2}$ are positive and high enough.

Proof. The observer error dynamics is given by:

$$
\begin{aligned}
& \dot{\tilde{\sigma}}=\tilde{\xi}(t)-K_{1} \Phi_{1}(\tilde{\sigma}) \\
& \dot{\tilde{\xi}}=-K_{2} \Phi_{2}(\tilde{\sigma})-h(t)
\end{aligned}
$$

Rewriting (15) in the following form, yields to:

$$
\begin{aligned}
& s_{1 i}=\tilde{\sigma}_{i} \\
& s_{2 i}=\tilde{\xi}(t)
\end{aligned}
$$


Then (15) can be rewritten in scalar form $(i=\overline{1,6})$ as:

$$
\begin{aligned}
& \dot{s}_{1 i}=-k_{1 i}\left[\mu_{1 i}\left|s_{1 i}\right|^{\frac{1}{2}} \operatorname{sgn}\left(s_{1 i}\right)+\mu_{2 i} s_{1 i}\right]+s_{2 i} \\
& \dot{s}_{2 i}=-k_{2 i}\left[\frac{1}{2} \mu_{1 i}^{2} \operatorname{sgn}\left(s_{1 i}\right)+\frac{3}{2} \mu_{1 i} \mu_{2 i}\left|s_{1 i}\right|^{\frac{1}{2}} \operatorname{sgn}\left(s_{1 i}\right)+\mu_{2 i}^{2} s_{1 i}\right]+h_{i}(t)
\end{aligned}
$$

Without loss of generality, we can represent the system (16) with simplified notation:

$$
\begin{aligned}
& \dot{s}_{1}=-k_{1}\left[\mu_{1}\left|s_{1}\right|^{\frac{1}{2}} \operatorname{sgn}\left(s_{1}\right)+\mu_{2} s_{1}\right]+s_{2} \\
& \dot{s}_{2}=-k_{2}\left[\frac{1}{2} \mu_{1}^{2} \operatorname{sgn}\left(s_{1}\right)+\frac{3}{2} \mu_{1} \mu_{2}\left|s_{1}\right|^{\frac{1}{2}} \operatorname{sgn}\left(s_{1}\right)+\mu_{2}^{2} s_{1}\right]+h(t)
\end{aligned}
$$

Noting that $\phi_{2}\left(s_{1}\right)=\phi_{1}^{\prime}\left(s_{1}\right) \phi_{1}\left(s_{1}\right)$, where $\phi_{1}^{\prime}\left(s_{1}\right)=\left(\mu_{1} \frac{1}{2\left|s_{1}\right|^{1 / 2}}+\mu_{2}\right)$, and selecting the vector $\zeta\left(s_{1}, s_{2}\right)=\left[\zeta_{1}, \zeta_{2}\right]^{T}=\left[\phi_{1}\left(s_{1}\right), s_{2}\right]^{T}$ and $\rho=\frac{h(t)}{\phi_{1}^{\prime}\left(s_{1}\right)}$, it is possible to rewrite the system (17) as follows:

$$
\dot{\zeta}\left(s_{1}, s_{2}\right)=\phi_{1}^{\prime}\left(s_{1}\right)[A \zeta+B \rho]
$$

where the matrices are defined as follows:

$$
A=\left[\begin{array}{cc}
-k_{1} & 1 \\
-k_{2} & 0
\end{array}\right], \quad B=\left[\begin{array}{l}
0 \\
1
\end{array}\right]
$$

Consider the Lyapunov candidate function as follows [28]:

$$
V=\zeta^{T} P \zeta
$$

where $P$ is a positive definite matrix which satisfies the Lyapunov equation:

$$
A^{T} P+P A=-Q
$$

where $Q$ is any given positive definite matrix.

Note that the proposed Lyapunov candidate function is a continuous, positive definite and differentiable function which satisfies the next form:

$$
\lambda_{\min }(P)\|\zeta\|_{2}^{2} \leq V(s) \leq \lambda_{\max }(P)\|\zeta\|_{2}^{2}
$$

Where $\lambda_{\min }(P)$ and $\lambda_{\max }(P)$ are the smallest and greatest eigenvalue of $P$, respectively. $\|\zeta\|_{2}^{2}=$ $\zeta_{1}^{2}+\zeta_{2}^{2}=\mu_{1}^{2}\left|s_{1}\right|+2 \mu_{1} \mu_{2}\left|s_{1}\right|^{\frac{3}{2}}+\mu_{2}^{2} s_{1}^{2}+s_{2}^{2}$ is the square of the Euclidean norm of $\zeta$ and noting that:

$$
\left|\phi\left(s_{1}\right)\right| \leq\|\zeta\|_{2} \leq \frac{V^{\frac{1}{2}}(\zeta)}{\lambda_{\min }^{\frac{1}{2}}(P)}
$$


Remark. For design reasons, it is important to note that the gain matrix A from (19) can be rewritten as:

$$
A=A_{0}-K_{0} C_{0}
$$

where:

$$
A_{0}=\left[\begin{array}{ll}
0 & 1 \\
0 & 0
\end{array}\right], K_{0}=\left[\begin{array}{l}
k_{1} \\
k_{2}
\end{array}\right] \quad, C_{0}=\left[\begin{array}{ll}
1 & 0
\end{array}\right]
$$


The time derivative of $V$ along the trajectories of the system is defined as follows:

$$
\begin{aligned}
& \dot{V}=2 \zeta^{T} P \dot{\zeta} \\
& =\phi_{1}^{\prime}\left(s_{1}\right)\left[\zeta^{T}\left(A^{T} P+P A\right) \zeta+\zeta^{T} P B \rho+\rho^{T} B^{T} P \zeta\right] \\
& =\phi_{1}^{\prime}\left(s_{1}\right)\left[\begin{array}{l}
\zeta \\
\rho
\end{array}\right]^{T}\left[\begin{array}{cc}
A^{T} P+P A & P B \\
B^{T} P & 0
\end{array}\right]\left[\begin{array}{l}
\zeta \\
\rho
\end{array}\right] \\
& \leq \phi_{1}^{\prime}\left(s_{1}\right)\left\{\left[\begin{array}{l}
\zeta \\
\rho
\end{array}\right]^{T}\left[\begin{array}{cc}
A^{T} P+P A & P B \\
B^{T} P & 0
\end{array}\right]\left[\begin{array}{l}
\zeta \\
\rho
\end{array}\right]+\omega(\rho, \zeta)\right\} \\
& =\phi_{1}^{\prime}\left(s_{1}\right)\left\{\left[\begin{array}{l}
\zeta \\
\rho
\end{array}\right]^{T}\left[\begin{array}{cc}
A^{T} P+P A+L^{2} C^{T} C & P B \\
B^{T} P & -1
\end{array}\right]\left[\begin{array}{l}
\zeta \\
\rho
\end{array}\right]\right\} \\
& =\phi_{1}^{\prime}\left(s_{1}\right)\left\{\left[\begin{array}{l}
\zeta \\
\rho
\end{array}\right]^{T}\left[\begin{array}{cc}
A^{T} P+P A+L^{2} C^{T} C+\bar{\alpha} P & P B \\
B^{T} P & -1
\end{array}\right]\left[\begin{array}{l}
\zeta \\
\rho
\end{array}\right]\right\} \\
& -\phi_{1}^{\prime}\left(s_{1}\right) \bar{\alpha} \zeta^{T} P \zeta \\
& =\phi_{1}^{\prime}\left(s_{1}\right)\{\left[\begin{array}{l}
\zeta \\
\rho
\end{array}\right]^{T} \underbrace{\left[\begin{array}{c|c}
-C_{0}^{T} K_{0}^{T} P-P K_{0} C_{0}+\bar{\alpha} P & P B \\
+A_{0}^{T} P+P A_{0}+L^{2} C^{T} C & \\
B^{T} P & -1
\end{array}\right]}_{W\left(K_{0}, P \mid \bar{\alpha}, L\right)}\left[\begin{array}{l}
\zeta \\
\rho
\end{array}\right]\} \\
& -\phi_{1}^{\prime}\left(s_{1}\right) \bar{\alpha} \zeta^{T} P \zeta \\
& =\phi_{1}^{\prime}\left(s_{1}\right)\left\{\left[\begin{array}{l}
\zeta \\
\rho
\end{array}\right]^{T} W\left(K_{0}, P \mid \bar{\alpha}, L\right)\left[\begin{array}{l}
\zeta \\
\rho
\end{array}\right]^{T}-\bar{\alpha} \zeta^{T} P \zeta\right\}
\end{aligned}
$$

Assuming that $K_{0}$ is selected in such way that exists $P>0$ and $\bar{\alpha}>0$ providing $W\left(K_{0}, P \mid \bar{\alpha}, L\right) \leq$ 0 . Then, the time derivative of $V$ can be expressed as follows:

$$
\begin{aligned}
\dot{V}(\zeta) & \leq-\bar{\alpha} \phi_{1}^{\prime}\left(s_{1}\right) \zeta^{T} P \zeta \\
& =-\mu_{1} \frac{\bar{\alpha}}{2\left|s_{1}\right|^{\frac{1}{2}}} V-\mu_{2} \bar{\alpha} V \\
& \leq-\frac{\mu_{1} \bar{\alpha} \lambda_{\min }^{\frac{1}{2}}(P)}{2} V^{\frac{1}{2}}-\mu_{2} \bar{\alpha} V
\end{aligned}
$$


The fact that the derivative of $V$ is definite negative is reached by selecting the positive gains $k_{1}$ and $k_{2}$ high enough to satisfy the condition $W\left(K_{0}, P \mid \bar{\alpha}, L\right) \leq 0$. Therefore, it can be concluded that the equilibrium point is reached in finite time from every initial condition.

Since the solution of its analog differential equation of 27p is given by:

$$
v(t)=\exp \left(-\mu_{2} \bar{\alpha} t\right)\left[v(0)^{\frac{1}{2}}+\frac{\mu_{1} \lambda_{\min }^{\frac{1}{2}}(P)}{2 \mu_{2}}\left[1-\exp \left(\frac{\mu_{2} \bar{\alpha}}{2} t\right)\right]\right]^{2}
$$

it follows that the solution converges in finite time to the origin at most after time $T$, which is computed as follows:

$$
T=\frac{2}{\mu_{2} \bar{\alpha}} \ln \left(\frac{2 \mu_{2}}{\mu_{1} \lambda_{\min }^{\frac{1}{2}}(P)} v(0)^{\frac{1}{2}}+1\right)
$$

Finally, using the comparison principle it can be stated that the observer internal states $(\hat{\sigma}, \hat{d})$ converge to $(\sigma, d)$ at most after a time given by [29].

Remark. The matrix $W\left(K_{0}, P \mid \alpha, L\right)<0$ is a Bilinear Matrix Inequality due the product of $P$ and $K_{0}$. In order to solve this problem as a Linear Matrix Inequality (LMI) it can be introduced the following matrix:

$$
Y=P K_{0}
$$

The matrix $W$ can be rewritten in the next form:

$$
W=\left[\begin{array}{c|c}
-C_{0}^{T} Y^{T}-Y C_{0}+\bar{\alpha} P & P B \\
A_{0}^{T} P+P A_{0}+L^{2} C^{T} C & \\
\hline B^{T} P & -1
\end{array}\right]
$$

This representation of $W$ can be seen as LMI on $P$ and $Y$. Note that it is needed to know the bound of the disturbance and a fixed positive constant value $\bar{\alpha}>0$ in order to solve the LMI (31) and be able to find the gains of the GSTA-ESO trough the following relationship:

$$
K_{0}=P^{-1} Y
$$

\section{B. Enhanced Nonlinear PD Controller Design}

In this section, a brief description of the enhanced NLPD (eNLPD) controller design is shown. Based on the proof of the Theorem 1, the GSTA-ESO estimates the disturbance term in a finite time. Then, the estimated measurement is inserted into the controller in order to compensate the 
Theorem 2. Let the AUV mathematical model with external disturbances be defined by equation (7). Introducing the disturbance estimation $\hat{d}(t)$ given by equations (14) into the following nonlinear PD controller

$$
\tau_{\eta}=\hat{M}_{\eta}(\eta) \ddot{\eta}_{d}+\hat{C}_{\eta}(\nu, \eta) \dot{\eta}_{d}+\hat{D}_{\eta}(\nu, \eta) \dot{\eta}_{d}-\hat{g}_{\eta}(\eta)-K_{p}(\cdot) e-K_{d}(\cdot) \dot{e}-\hat{M}_{\eta}(\eta) \hat{d}-\hat{K} S g n(\dot{e})
$$

where $e(t)=\left[e_{1}(t), e_{2}(t), \cdots, e_{6}(t)\right]^{T}=\eta(t)-\eta_{d}(t)$ is the error signal, $\dot{e}(t)$ its time derivative, and the desired trajectory is defined as $\eta_{d}(t)=\left[x_{d}(t), y_{d}(t), z_{d}(t), \phi_{d}(t), \theta_{d}(t), \psi_{d}(t)\right]^{T}$. $\hat{K}$ is a positive constant, and the vector $\operatorname{Sgn}(\dot{e})=\left[\operatorname{sgn}\left(\dot{e}_{1}(t)\right), \operatorname{sgn}\left(\dot{e}_{2}(t)\right), \cdots, \operatorname{sgn}\left(\dot{e}_{6}(t)\right)\right]$. The gain matrices $K_{p}(\cdot)$ and $K_{d}(\cdot)$ have the following structure:

$$
\begin{aligned}
K_{p}(\cdot)= & {\left[\begin{array}{cccc}
k_{p 1}(\cdot) & 0 & \cdots & 0 \\
0 & k_{p 2}(\cdot) & \cdots & 0 \\
\vdots & \vdots & \ddots & \vdots \\
0 & 0 & \cdots & k_{p n}(\cdot)
\end{array}\right]>0 } \\
K_{d}(\cdot)= & {\left[\begin{array}{cccc}
k_{d 1}(\cdot) & 0 & \cdots & 0 \\
0 & k_{d 2}(\cdot) & \cdots & 0 \\
\vdots & \vdots & \ddots & \vdots \\
0 & 0 & \cdots & k_{d n}(\cdot)
\end{array}\right]>0 }
\end{aligned}
$$

and asymptotically stabilize the system (7) if $k_{p j}(\cdot)$ and $k_{d j}(\cdot)$ are defined as:

$$
\begin{aligned}
k_{p j}(\cdot) & = \begin{cases}b_{p j}\left|e_{j}(t)\right|^{\left(\mu_{p j}-1\right)} & \text { if }\left|e_{j}(t)\right|>d_{p j} \\
b_{p j} d_{p j}^{\left(\mu_{p j}-1\right)} & \text { if }\left|e_{j}(t)\right| \leq d_{p j}\end{cases} \\
k_{d j}(\cdot) & = \begin{cases}b_{d j}\left|\dot{e}_{j}(t)\right|^{\left(\mu_{d j}-1\right)} & \text { if }\left|\dot{e}_{j}(t)\right|>d_{d j} \\
b_{d j} d_{d j}^{\left(\mu_{d j}-1\right)} & \text { if }\left|\dot{e}_{j}(t)\right| \leq d_{d j}\end{cases} \\
\forall \mu_{p j}, \mu_{d j} \in[0,1] . &
\end{aligned}
$$

with the positive constants $b_{p j}, b_{d j}, d_{p j}$, and $d_{d j}$. 
Proof. Injecting the control law (33) into the system (7), the closed-loop system is given by:

$$
\frac{d}{d t}\left[\begin{array}{l}
e \\
\dot{e}
\end{array}\right]=\left[\begin{array}{c}
\dot{e} \\
-\hat{M}_{\eta}(\eta)^{-1}\left[\left[\hat{C}_{\eta}(\nu, \eta)+\hat{D}_{\eta}(\nu, \eta)+K_{d}(\cdot)\right] \dot{e}+K_{p}(\cdot) e+\hat{K} \operatorname{Sgn}(\dot{e})\right]-\hat{d}(t)+d(t)
\end{array}\right]
$$

Considering the following Lyapunov candidate function:

$$
V(e, \dot{e})=\frac{1}{2} \dot{e}^{T} \hat{M}_{\eta}(\eta) \dot{e}+\int_{0}^{e} \varrho^{T} K_{p}(\varrho) d \varrho+\frac{1}{2 \beta} \tilde{K}^{2}
$$

where

$$
\int_{0}^{e} \varrho^{T} K_{p}(\varrho) d \varrho=\int_{0}^{e_{1}} \varrho^{T} K_{p}(\varrho) d \varrho+\int_{0}^{e_{2}} \varrho^{T} K_{p}(\varrho) d \varrho+\cdots+\int_{0}^{e_{6}} \varrho^{T} K_{p}(\varrho) d \varrho
$$

with the parameter estimation error defined as $\tilde{K}=\hat{K}-K$, and $\beta$ is a positive constant.

This function is positive definite and radially unbounded (see [7] for more details). The time derivative of the Lyapunov candidate function is given by:

$$
\dot{V}(e, \dot{e})=\dot{e}^{T} \hat{M}_{\eta}(\eta) \ddot{e}+\frac{1}{2} \dot{e}^{T} \dot{\hat{M}}_{\eta}(\eta) \dot{e}+e^{T} K_{p}(\cdot) \dot{e}+\frac{1}{\beta} \tilde{K} \dot{\hat{K}}
$$

Considering that the GSTA-ESO converges to the disturbance dynamics in finite time, it is reasonable to assume that $\|d(t)-\hat{d}(t)\| \leq K$ with the unknown constant $K>0$. The constant $K$ was obtained through the following adaption law

$$
\dot{\hat{K}}=\beta\|\dot{e}\|
$$

Substituting the error dynamics (38) into the time derivative of $V$ and considering the Assumption 2 and equation 42 , it can be noticed the following:

$$
\begin{aligned}
\dot{V}(e, \dot{e}) & =-\dot{e}^{T}\left[\hat{D}_{\eta}(\nu, \eta)+K_{d}(\cdot)\right] \dot{e}+\dot{e}^{T}[d(t)-\hat{d}(t)]-\hat{K} \dot{e}^{T} \operatorname{Sgn}(\dot{e})+\frac{1}{\beta} \tilde{K} \dot{\hat{K}} \\
& =-\dot{e}^{T}\left[\hat{D}_{\eta}(\nu, \eta)+K_{d}(\cdot)\right] \dot{e}+\dot{e}^{T}[d(t)-\hat{d}(t)]-\hat{K} \sum_{i=0}^{6}\left|\dot{e}_{i}\right|+\frac{1}{\beta} \tilde{K} \dot{\hat{K}} \\
& \leq-\lambda_{\min }\left(\hat{D}_{\eta}(\nu, \eta)+K_{d}(\cdot)\right)\|\dot{e}\|^{2}+K\|\dot{e}\|-\hat{K}\|\dot{e}\|+\tilde{K}\|\dot{e}\| \\
& =\leq-\lambda_{\min }\left(\hat{D}_{\eta}(\nu, \eta)+K_{d}(\cdot)\right)\|\dot{e}\|^{2}
\end{aligned}
$$

From the controller construction stated before, the gain matrix is $K_{d}(\cdot)>0$ by design and the damping matrix fulfills $\hat{D}_{\eta}(\nu, \eta)>0$ [2]. Then, the function $\dot{V}$ is negative semi-definite. Finally, applying the Krasovskii-Lasalle's theorem we can conclude that the equilibrium point is asymptotically stable [7]. 


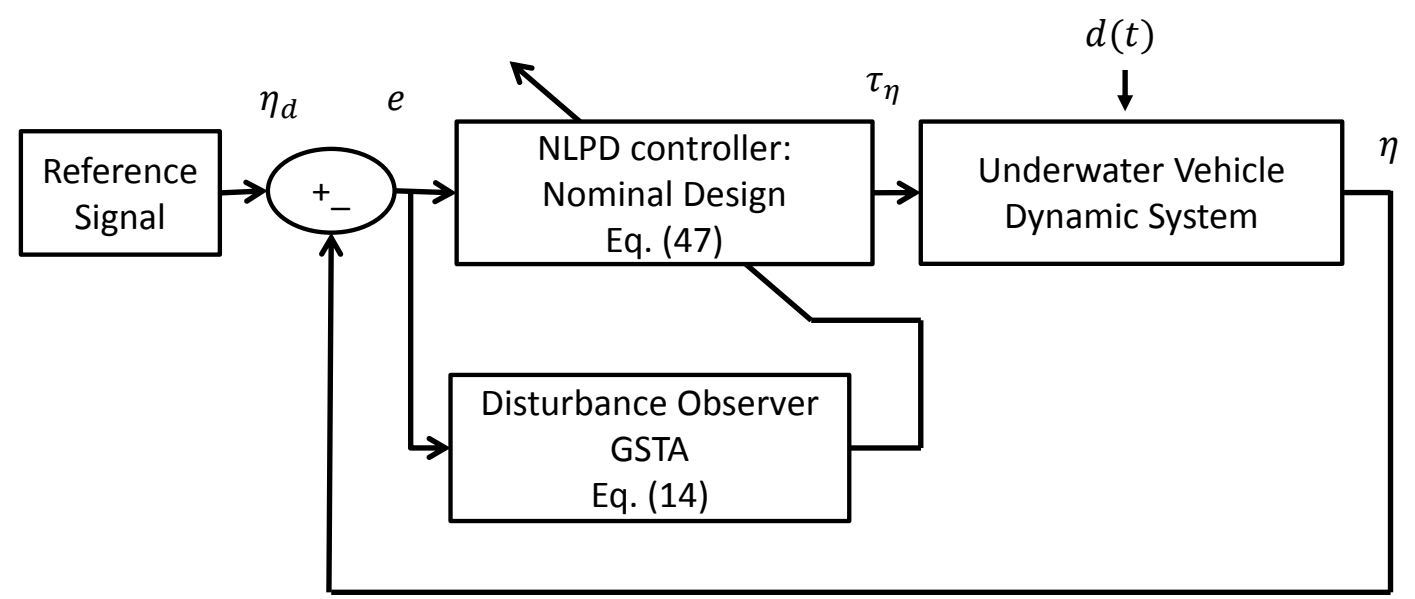

Fig. 2. Proposed controller/observer scheme for trajectory tracking for underwater vehicles.

Remark. In the experimental part of this work, the performance of the developed controller law given by equation (33) is compared against the control proposed in [7]:

$$
\tau=J^{T}(\eta)\left[\hat{M}_{\eta}(\eta) \ddot{\eta}_{d}+\hat{C}_{\eta}(\nu, \eta) \dot{\eta}_{d}+\hat{D}_{\eta}(\nu, \eta) \dot{\eta}_{d}-\hat{g}_{\eta}(\eta)-K_{p}(\cdot) e-K_{d}(\cdot) \dot{e}\right]
$$

Remark. To provide a better understand of the proposed control/observer, the block diagram of the scheme is illustrated in Fig. 2.

\section{REAL-TIME EXPERIMENT RESULTS}

To demonstrate the practical feasibility of the developed controller, we applied the control algorithm to Leonard (illustration of Figure 3), which is an underwater vehicle developed at the LIRMM (University of Montpellier / CNRS, France). The Leonard is a tethered underwater vehicle which measures $75 \times 55 \times 45 \mathrm{~cm}$ and $28 \mathrm{~kg}$ in weight. The propulsion system of this vehicle consists of six independent thrusters to obtain a full actuated system.

The experimental platform consists of a ROV controlled by a laptop computer, with CPU Intel Core i7-3520M 2.9 GHz, 8GB of RAM. The machine runs under Windows 7 operating system, and the control software is developed using Visual C++ 2010. The computer receives the data 
from the robot's sensors (depth, attitude), computes the control laws and sends input signals to the propellers. These actuators are controlled by Syren 25 Motor Drives. The main features of this vehicle are summarized in Table $\mathrm{I}$ and the estimated parameters of the Leonard underwater vehicle are shown in Table $\amalg$.

\section{TABLE I}

MAIN FEATURES OF THE UNDERWATER VEHICLE

\begin{tabular}{ll}
\hline Mass & $28 \mathrm{~kg}$ \\
Dimensions & $75 \times 55 \times 45 \mathrm{~cm}$ \\
Maximal depth & $100 \mathrm{~m}$ \\
Thrusters & 6 Seabotix BTD150 \\
Power & $48 \mathrm{~V}-600 \mathrm{~W}$ \\
Attitude Sensor & Sparkfun Arduimu V3 \\
& Invensense MPU-6000 MEMS 3-axis gyro \\
& and accelerometer \\
& 3-axis I2C magnetometer HMC-5883L \\
& Atmega328 microprocessor \\
& Pacific Co. VPC-895A \\
Camera & CCD1/3 PAL-25-fps \\
& Pressure Sensor Breakout-MS5803-14BA \\
Depth sensor & 40 ms \\
Sampling period & Dell Latitude E6230- Intel Core i7 -2.9 GHz \\
Surface computer & Windows 7 Professional 64 bits \\
& Microsoft Visual C++ 2010 \\
Tether length & 150 m \\
\hline
\end{tabular}

TABLE II

ESTIMATED PARAMETERS OF LEONARD UNDERWATER VEHICLE

\begin{tabular}{ll}
\hline$\hat{M}_{\eta}(\eta)$ & $\operatorname{diag}\left(28[\mathrm{~kg}], 28[\mathrm{~kg}], 28[\mathrm{~kg}], 0.5\left[\mathrm{~kg} \cdot \mathrm{m}^{2}\right], 2\left[\mathrm{~kg} \cdot \mathrm{m}^{2}\right], 0.65\left[\mathrm{~kg} \cdot \mathrm{m}^{2}\right]\right)$ \\
$\hat{D}_{\eta}(\nu, \eta)$ & $\operatorname{diag}\left(30\left[\frac{\mathrm{N} \cdot \mathrm{s}}{\mathrm{m}}\right], 40\left[\frac{\mathrm{N} \cdot \mathrm{s}}{\mathrm{m}}\right], 60\left[\frac{\mathrm{N} \cdot \mathrm{s}}{\mathrm{m}}\right], 1.4\left[\frac{\mathrm{N} \cdot \mathrm{s}}{\mathrm{rad}}\right], 2.5\left[\frac{\mathrm{N} \cdot \mathrm{s}}{\mathrm{rad}}\right], 2.9\left[\frac{\mathrm{N} \cdot \mathrm{s}}{\mathrm{rad}}\right]\right)$ \\
$\hat{g}_{\eta}(\eta)$ & {$[0[N],-12[N],-12[N],-0.1[N],-0.1[N], 0[N]]^{T}$} \\
\hline
\end{tabular}


The control algorithm was experimentally tested in a $4 \times 4 \times 1.2 \mathrm{~m}$ pool of the LIRMM. Although the proposed control law given by Eq. (33) is designed for the whole system of six degrees of freedom, the real-time experiments conducted in this work are focused on the depth and Yaw dynamics. The main goal of the designed controller is to track robustly the desired reference trajectory in depth and yaw in the presence of parameter uncertainties and external disturbances.

The experimental results proposed hereafter have been conducted through the implementation of the proposed controllers on the of Leonard underwater vehicle. The real-time experiments are available at: https://www.youtube.com/watch?v=cZ8c53K7qkU,

\section{A. Proposed Experimental Scenarios}

To test the robustness of the proposed controllers, we propose a set of different scenarios. The main idea of this experiments is to show the improvement of adding the disturbance observer to the nominal controller design. The following three cases have been considered, namely:

(i) Scenario 1: Nominal case.

In this scenario, the robot follows a predefined desired trajectory in depth and yaw in the absence of external disturbances. During this test, the controller's gains are adjusted to obtain the best tracking. These gains remain unchanged during the rest of the experiments.

(ii) Scenario 2: Robustness towards parametric uncertainties

In this test, the buoyancy and damping of the vehicle are modified to test the effectiveness of the controller and its robustness towards parametric uncertainties.

(iii) Scenario 3: External disturbances rejection.

This test is inspired by a more realistic scenario, where the vehicle has the task of loading an object and when reaching a certain depth, dropping that object. In this test, it is possible to see a sudden change in vehicle's weight and how it affects the controller performance.

\section{B. Procedure to tune the gains of the proposed controller and disturbance observer}

It is important to highlight that in the whole set of experiments, all controllers were tuned heuristically but always considering the constraints given by the stability proofs shown above. For example, the NLPD controller was tuned under procedure given in work [7]. For the tuning of the GSTA-ESO, it is worth to note that from equation (14), the gain $K_{2}$ is directly responsible 
for estimating the disturbance while the gain $K_{1}$ adjust the error of the auxiliary variable. The experimental procedure is enclosed as follows:

1) We set the gains $K_{2}=0.0001$ and $\Lambda=1$. Then, the gain $K_{1}$ is increased until the behavior of the variable $\hat{\sigma}$ is close to the auxiliary variable $\sigma$ which depends on sensor's measurements, so it is entirely known.

2) When the behavior of $\hat{\sigma}$ is visually similar to $\sigma$, then the gain $K_{2}$ is increased until the controller's behavior in the steady state starts to oscillate.

3) The gain $\Lambda$ is responsible for the converge speed. It can be increased to a high value, but there is a trade-off between this gain and the amplitude of the chattering effect on the estimation of the disturbance.

Due the sampling period and to prevent the chattering effect in the control signal of the GSTA, it is suggested to keep the gain $K_{2}$ in a small value. Now, the tuning of the adaptive law $\hat{K}$ is obtained through the integration of Equation (42). From this equation, one can notice that its value depends of the norm of the time derivative of the error and the gain $\beta$. Then, in order to minimize the chattering effect due to the signum function into the control law, the gain $\beta$ is suggested to be kept in a small value. In the real-time experiments, this gain is considered as $\beta \rightarrow 0$ and therefore $\hat{K} \rightarrow 0$. After tuning the controllers for a constant reference, the control laws were tested for a trajectory tracking task without considering external disturbances, where the values of the gains were improved until reach a good performance and can be seen in Tables III and IV. Finally, the gains found with the previous procedure were unchanged during the robustness tests.

TABLE III

NLPD CONTROL GAINS USED IN REAL-TIME EXPERIMENTS

\begin{tabular}{llll}
\hline Depth & $b_{p 3}=20$ & $d_{p 3}=0.05$ & $\mu_{p 3}=0.1$ \\
& $b_{p 3}=13$ & $d_{p 3}=0.25$ & $\mu_{p 3}=0.2$ \\
Yaw & $b_{p 3}=4.5$ & $d_{p 3}=0.015$ & $\mu_{p 3}=0.2$ \\
& $b_{p 3}=0.2$ & $d_{p 3}=0.15$ & $\mu_{p 3}=0.2$ \\
\hline
\end{tabular}


TABLE IV

DISTURBANCE OBSERVER GAINS USED IN REAL-TIME EXPERIMENTS

\begin{tabular}{cccc}
\hline Depth & $k_{13}=0.7$ & $k_{23}=0.5$ & $\lambda_{3}=2.0$ \\
Yaw & $k_{16}=0.7$ & $k_{26}=0.5$ & $\lambda_{6}=2.0$ \\
\hline
\end{tabular}

\section{Scenario 1: Control in nominal conditions}

The upper plot of Figure 5 shows the depth and yaw controller's performance during the first case. In this experiment, the vehicle follows a predefined trajectory in depth going from the surface to a maximal depth of $30 \mathrm{~cm}$, where the vehicle remains stable for 20 seconds and finally reaches $20 \mathrm{~cm}$ and hovers until the trial ends. At the same time, the vehicle turns from its initial position to 60 degrees in 6 seconds. Then, the AUV remains stable in that position for 20 seconds. Finally, the robot goes to -60 degrees and stay there until the test ends. In this case, it can be noticed that the eNLPD scheme (red line) has a behavior visually similar to the NLPD controller (blue line). Both controllers take a short lapse of time (less than 5 seconds) to converge to the reference trajectory with a slight tracking error as seen in the error plot at the middle of Figure 5 and can be confirmed through numerical data of Root Mean Square Error (RMSE), which is given in Table $\mathrm{V}$. It is worth to note from Table $\mathrm{V}$ the superior performance of the eNLPD over the NLPD design. Finally, the evolution of the control inputs is displayed at the bottom of Figure 5

In the upper part of Figure 8 the estimated disturbance signal made by the GSTA-ESO during the real-time experiment is shown. Note that, from Figure 8, the estimated disturbance signal captured by the observer can be explained by the modeling errors in the system parameters (damping matrix or estimated buoyancy) used in the control law (33).

Finally, in order to estimate the energy consumption in the trajectory tracking test, the integral of control inputs is computed as follows:

$$
I N T=\int_{t_{1}}^{t_{2}}|\tau(t)| d t
$$

where $t_{1}=3 \mathrm{~s}$ and $t_{2}=50 \mathrm{~s}$. The estimated values for the integral are listed in Table VI. To compute the energy consumption for trajectory tracking for depth and yaw dynamics for both 
NLPD and eNLPD controllers, we need to divide the $I N T_{z}$ and $I N T_{\psi}$ for each methodology as follows:

$$
\frac{567}{557}=1.01 \quad \frac{28}{25}=1.12
$$

This means that energy consumption for trajectory tracking in depth, using the NLPD controller, is 1.01 times the energy consumption using the eNLPD control. While energy consumption for trajectory tracking in heading, using the eNLPD controller, is 1.12 times the energy consumption using the NLPD controller. In brief, the energy consumption is nearly the same for the eNLPD for tracking in depth but is highest for the tracking in heading.

\section{Scenario 2: Robustness towards parameter's uncertainties}

To evaluate the robustness of the proposed controller against parametric uncertainties, we changed the buoyancy of the vehicle by fixing two floaters to both sides of the vehicle, thus increasing the buoyancy by $+100 \%$. To modify the damping of the AUV, we attached a large rigid sheet of plastic that has a dimension of $45 \times 10 \mathrm{~cm}$ on one side of the submarine, increasing the rotational damping along z by approximately $90 \%$ (as illustrated in Figure 3 .

The AUV tracking trajectory for depth and yaw motion applying NLPD (blue line) and eNLPD (red line) controllers is shown on the top of Figure 6. From Figure 6, it is observed that the NLPD scheme is not able to compensate the high persistent parameter uncertainty on heave motion. In fact, the controller behavior is degraded and has an offset of $0.03 \mathrm{~m}$ with respect to the desired trajectory. On the other hand, the improvement of the eNLPD algorithm over the NLPD nominal design clearly appears. The disturbance observer action is capable of compensating the added buoyancy minimizing the steady-state error to a RMSE value of $0.0025 \mathrm{~m}$ during the depth tracking test. As expected, the eNLPD takes a short lapse of time to converge to the reference depth trajectory. This is due to the fact that the vehicle needs more energy to overcome the added buoyant force. In the meantime, although both controllers follow the yaw reference signal, the eNLPD does not improve the behavior of the NLPD control. Indeed, there is an undesirable effect when the vehicle turns, and this overshoot can be due to the selected high gain for the yaw disturbance observer.

The evolution of the tracking errors is shown in the middle of Figure 6. From this figure, it is possible to observe the impact of the disturbances. The error increases when the vehicle's depth changes or when it turns. The Table $\mathrm{V}$ shows the RMSE for both controllers. Finally, in 
the bottom of Figure 6, the evolution of the controller's inputs is shown. The eNLPD shows a slight increase of the energy to obtain a fast compensation of the disturbance effect during the first 5 seconds of the test. After that short period of time, the evolution of the eNLPD control inputs remains similar to the NLPD scheme.

In the middle of Figure 8 the estimated disturbance through the GSTA-ESO is displayed for the depth and yaw tracking. One can notice the impact of the persistent disturbance on depth motion due to the added extra buoyancy (see left figure). The observer tries to compensate this vertical disturbance with a constant signal. Meanwhile, the estimated disturbance on yaw motion has an offset at the beginning of the test. After, a peak appears due to the increased damping when the vehicle turns.

Finally, based on the results displayed in Table $\mathrm{VI}$, the quotients between $I N T_{z}$ and $I N T_{\psi}$ from the robustness towards parameter's uncertainties test are:

$$
\frac{1099}{1090}=1.008 \quad \frac{61}{51}=1.196
$$

This means that energy consumption for trajectory tracking in depth for both controllers are almost the same amount of energy. While energy consumption for trajectory tracking in heading, using the eNLPD controller, is 1.196 times the energy consumption using the NLPD control. Again, the eNLPD has nearly the same performance as the NLPD in terms of energy consumption for the tracking in depth.

Remark. From Figure 3 one can notice that the tether of the vehicle may affect the underwater robot motion. However, the ballast due to the tether can be seen as a non modeled dynamics, and the disturbance observer will counteract this external influence as one can notice from the experimental results.

\section{E. Scenario 3: Robustness towards external disturbances}

In some applications, AUV's are equipped with robotic manipulators which allow to carry or manipulate objects and take them to a specific depth or pick them up from the ocean floor to transport them to the surface. This scenario is inspired by that practical application, to simulate a mission where the robot carries a load. A metallic $1 \mathrm{~kg}$ block of was tied to the submarine with a $20 \mathrm{~cm}$-long line. In this test, the maximal depth was set to $40 \mathrm{~cm}$. As the maximum depth of the basin is $50 \mathrm{~cm}$, the robot will be suddenly disturbed when it reaches 30 centimeters, 
because the metallic block will touch the floor, thus suddenly canceling its weight's effect. The disturbance will be acting on the robot until it starts to emerge and it reaches $30 \mathrm{~cm}$, the action of the extra weight will influence the trajectory of the submarine again (as illustrated in Figure 4). This simulates both the sudden release and recovery of a load by the robot.

The results of the controller's performance in the robustness test against external disturbances are shown in the top of Figure 7 In this test, the yaw motion remained unperturbed. From the results displayed on the right side of Figure 7, the yaw motion behavior for the trajectory tracking test did not suffer of any change and remained similar to the Nominal case. Meanwhile, regarding depth tracking, due to the added extra weight, the submarine initial position had changed to $30 \mathrm{~cm}$ deep. When the test begins, the robot converges to the desired trajectory in about 5 seconds for the eNLPD algorithm and 7 seconds in the case of the NLPD control. In the 8th second, the weight of the vehicle suddenly changes and one can see that both controllers compensate the effect of the disturbance some seconds later. When the vehicle comes back up, the extra weight acts on the submarine degrading the trajectory tracking. As shown in Figure 7 , the NLPD controller is not capable of compensating the weight disturbance showing a constant steady-state error of approximately $10 \mathrm{~cm}$. Again, the eNLPD shows superior performance over NLPD compensating the persistent vertical disturbance and drastically reducing the steady-state error in depth.

The error plots are displayed in the middle of Figure 7 while the numerical value of the RMSE is given in Table $\mathrm{V}$. The evolution of the control inputs versus time is displayed at the bottom of Figure 7. At the end of the test, there is an undesirable chattering effect, but its amplitude decreases as time increased.

The estimated disturbance of the depth and yaw tracking controllers is shown at the bottom of Figure 8. From the left side of Figure 8, it can be observed the influence of the extra weight disturbance at the beginning and the end of the plot. Visually, the shape of the yaw disturbance signal is almost the same as in the nominal case.

Finally, from the results displayed in Table $\overline{\mathrm{VI}}$, the quotients between $I N T_{z}$ and $I N T_{\psi}$ from the robustness towards external disturbances test are:

$$
\frac{375}{361}=1.04 \quad \frac{33}{32}=1.03
$$

This means that energy consumption for trajectory tracking in depth, using the eNLPD controller, is 1.04 times the energy consumption using the NLPD control. While energy consumption for 


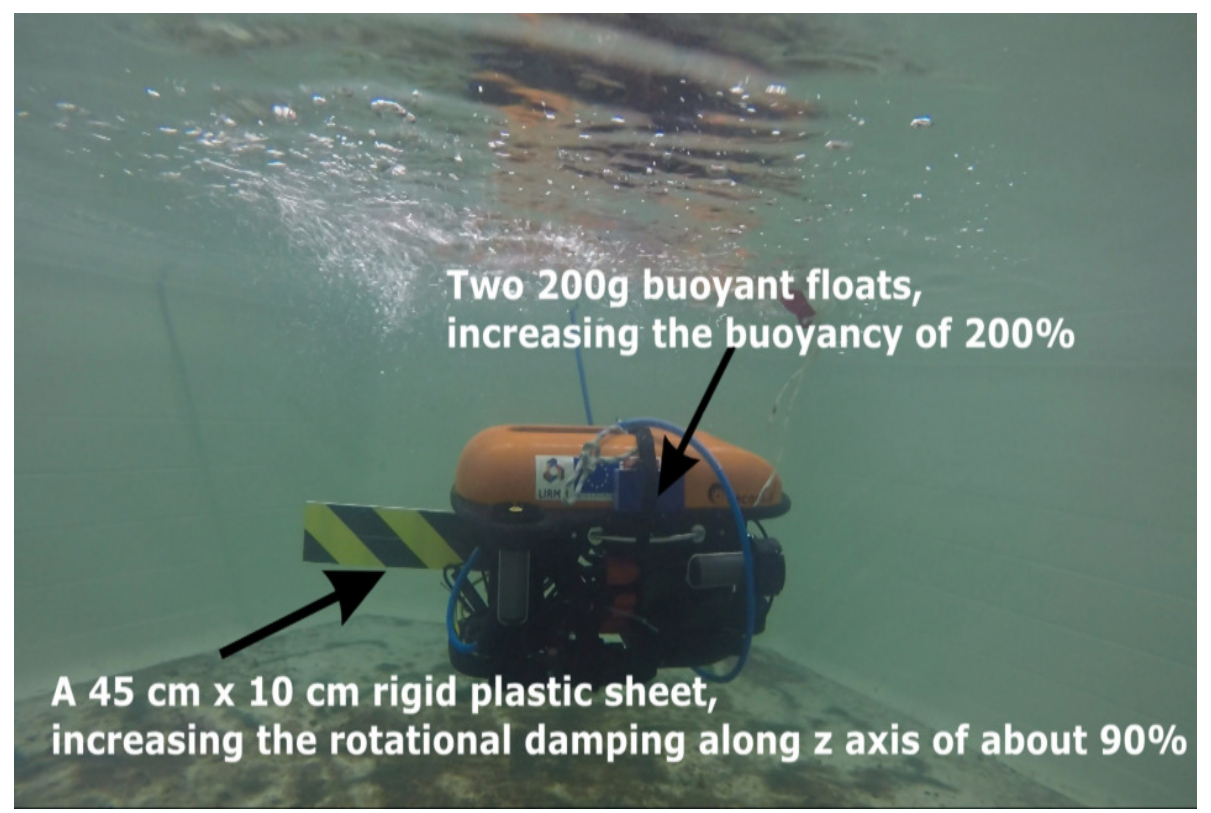

Fig. 3. Leonard underwater vehicle with the added two buoyant floats and a rigid plastic sheet, which will increase the buoyancy force and damping along $\mathrm{z}$ axis.

trajectory tracking in heading, using the eNLPD controller, is 1.03 times the energy consumption using the NLPD method.

TABLE V

RoOT MEAN SQUARE ERROR FOR NLPD AND ENLPD DESIGN.

\begin{tabular}{lcccc}
\hline \multirow{2}{*}{ Case } & \multicolumn{2}{c}{ NLPD } & \multicolumn{2}{c}{ eNLPD } \\
\cline { 2 - 5 } & $R M S E_{z}[m]$ & $R M S E_{\psi}[\mathrm{deg}]$ & $R M E_{z}[\mathrm{~m}]$ & $R M S E_{\psi}[\mathrm{deg}]$ \\
\hline Nominal & 0.0023 & 0.0265 & 0.00001 & 0.0175 \\
Parametric & 0.0374 & 0.3371 & 0.0025 & 0.3484 \\
Uncertainties & & & & \\
External & 0.0522 & 0.0571 & 0.0125 & 0.0135 \\
Disturbances & & & & \\
\hline
\end{tabular}




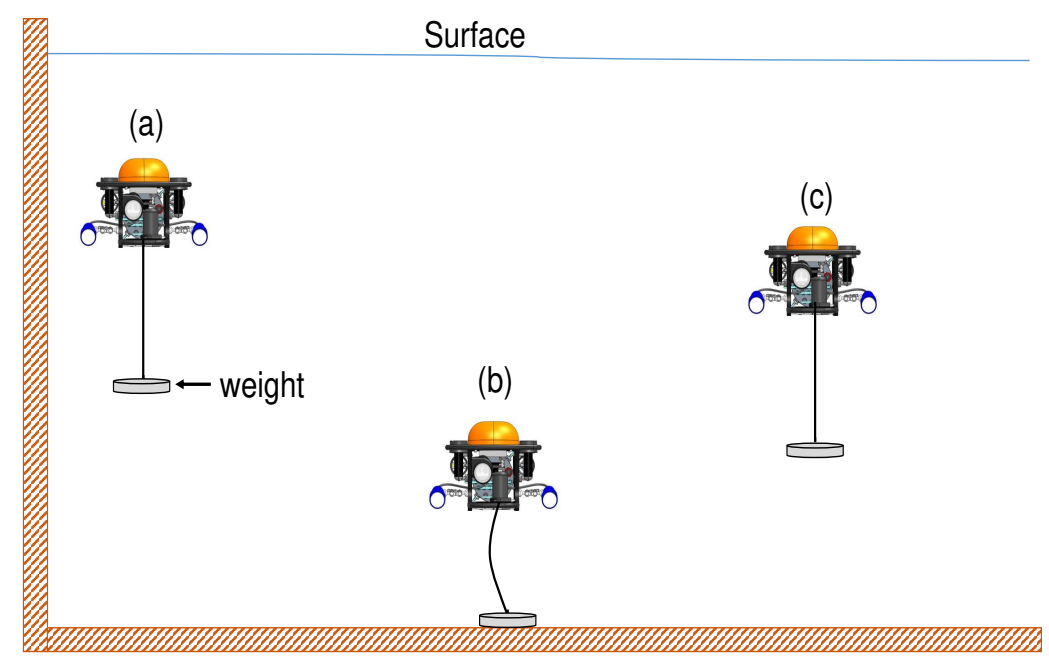

Fig. 4. Description of the test of the controller's robustness towards external disturbances. A $1 \mathrm{~kg}$ load is attached to the robot as shown in (a). When the robot reaches $30 \mathrm{~cm}$, the influence of the weight disappears (b). Finally, the robot comes up again and the influence of the weight acts again on the robot (c).

TABLE VI

INTEGRAL CONTROL OF INPUTS FOR NLPD AND ENLPD DESIGN.

\begin{tabular}{lcccc}
\hline \multirow{2}{*}{ Case } & \multicolumn{2}{c}{ NLPD } & \multicolumn{2}{c}{ eNLPD } \\
\cline { 2 - 5 } & $I N T_{z}$ & $I N T_{\psi}$ & $I N T_{z}$ & $I N T_{\psi}$ \\
\hline Nominal & 567 & 25 & 557 & 28 \\
Parametric & 1099 & 51 & 1090 & 61 \\
$\begin{array}{l}\text { Uncertainties } \\
\text { External }\end{array}$ & 361 & 32 & 375 & 33 \\
Disturbances & & & & \\
\hline
\end{tabular}

V. CONCLUSION

In this paper, an enhanced nonlinear PD controller for trajectory tracking of an AUV has been proposed. The nominal nonlinear PD controller design was improved by adding a disturbance observer based on high order sliding mode control, namely Generalized Super-Twisting Algorithm. The stability analysis for the resulting closed-loop system for trajectory tracking has been 
addressed. The proposed controller has been implemented for trajectory tracking in-depth and yaw motions with the Leonard underwater vehicle and has been compared to the nominal design. The real-time experiment's results demonstrate the effectiveness, robustness, and improvement of the proposed controller towards uncertainties on the parameters of the system (damping and buoyancy changes) and external disturbances. The future work will consist in implementing the adaptive version of the disturbance observer to obtain an auto-adjustable algorithm which will be able to reject bounded external disturbances effectively. Also, as future work, is mandatory to include a parametric sensitivity analysis to complete the robustness analysis for the proposed controller/observer scheme.

\section{ACKNOWLEDGMENT}

The authors would like to express their gratitude to the anonymous reviewers for the comments to the improvement of the manuscript. The authors thank CONACYT for the scholarship grant (490978). 

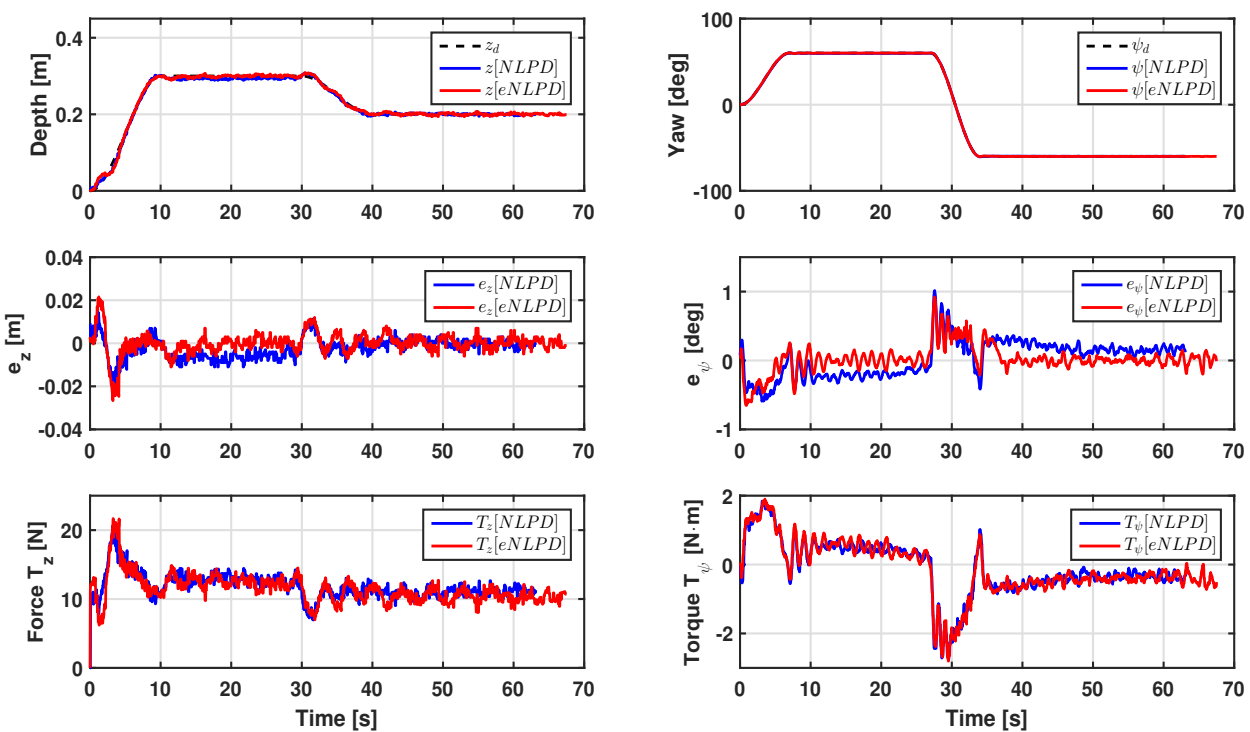

Fig. 5. Comparison of NLPD and eNLPD controllers for the depth and yaw tracking trajectory task in the nominal case.
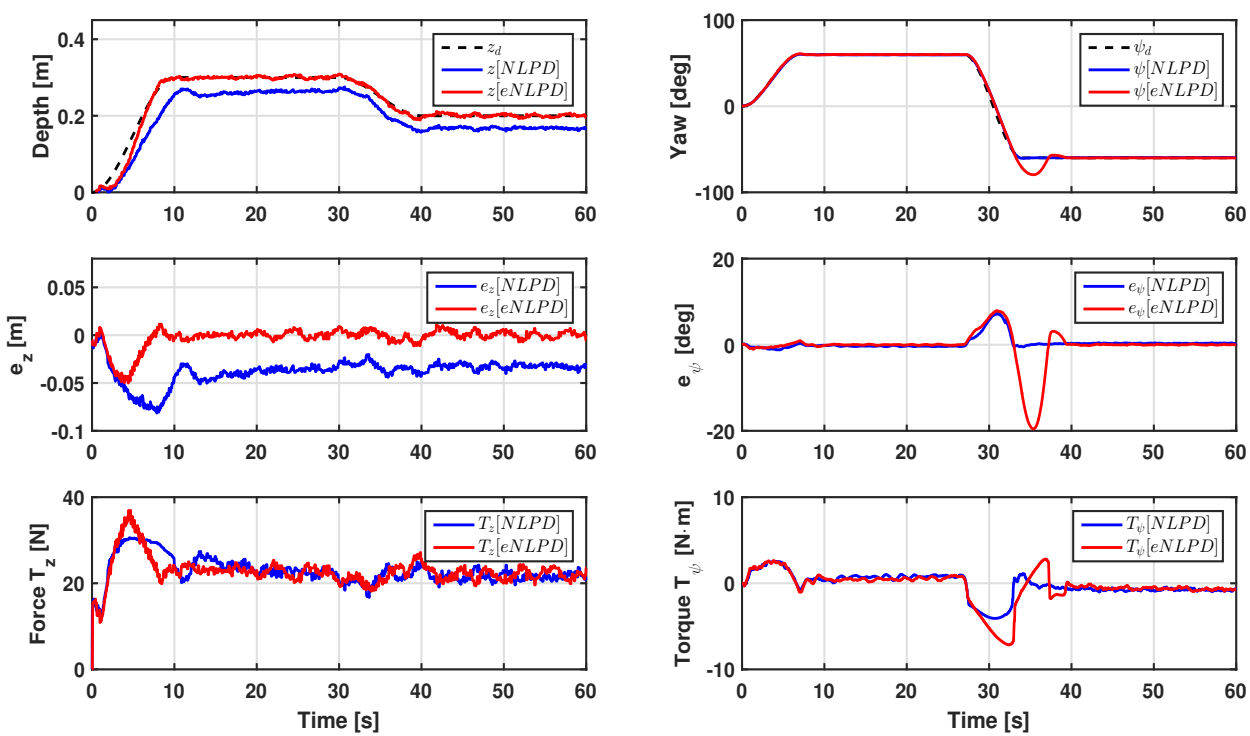

Fig. 6. Robustness of the NLPD and eNLPD controllers behavior towards parametric uncertainties. The floatability of the submarine was increased $+100 \%$ while the damping along $\mathrm{z}$-axis was modify up to $90 \%$ respect the nominal case. 

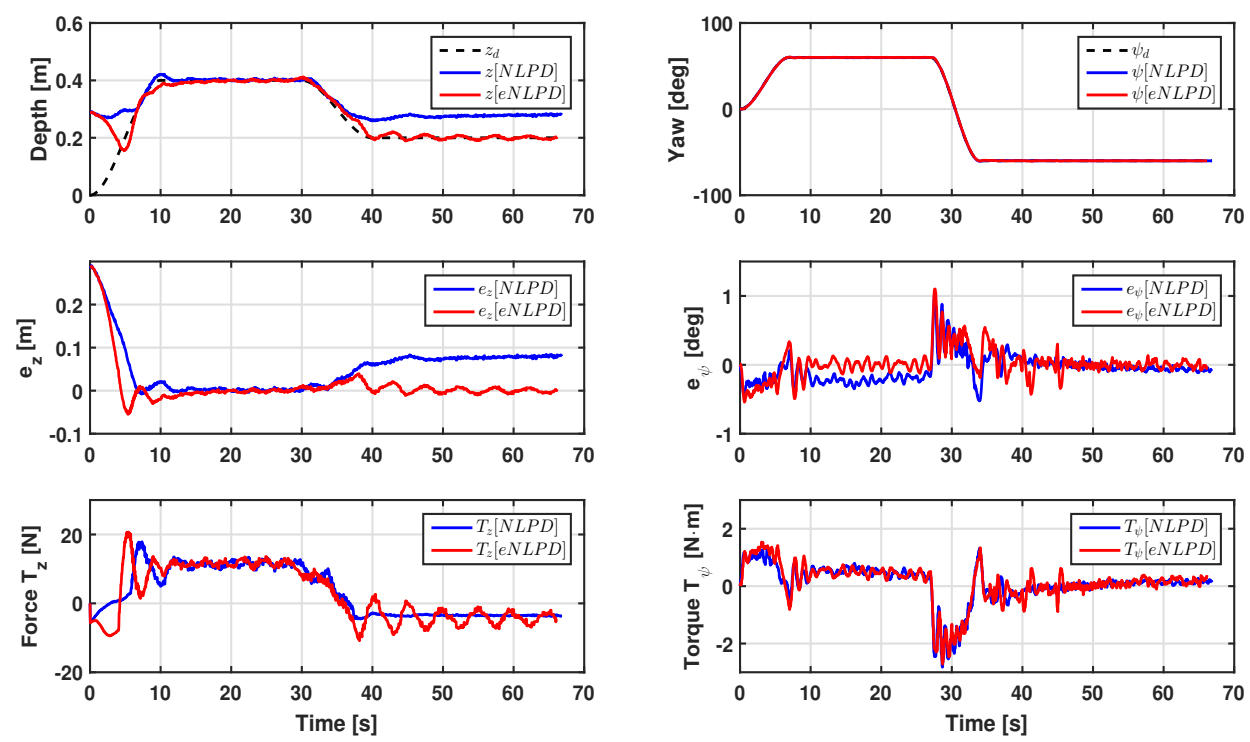

Fig. 7. Robustness of the NLPD and eNLPD controllers evolution towards external disturbances: Release and recovery of a load.
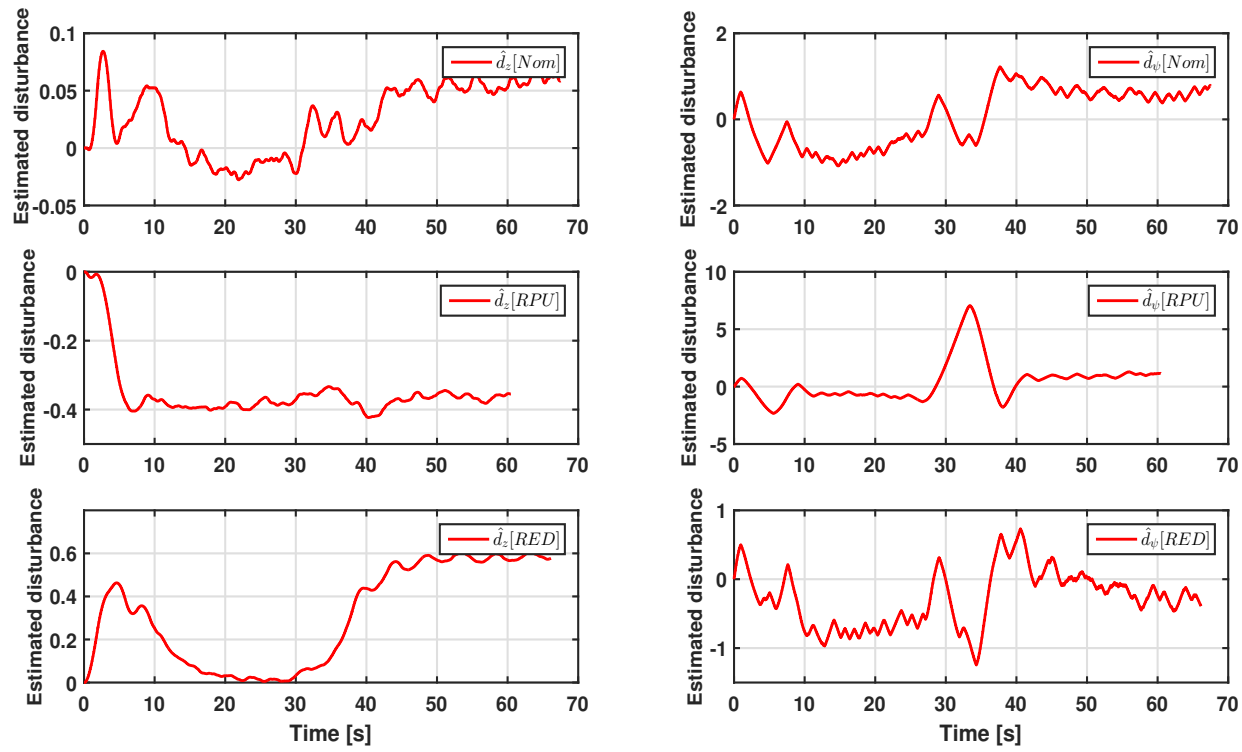

Fig. 8. Estimated disturbance for the trajectory tracking in depth and yaw motions. The disturbance estimation in the nominal case is shown in the upper part. In the middle of this figure is displayed the robustness tests towards parameter uncertainties. Disturbance observation during the external disturbances rejection test is shown at the bottom. 


\section{REFERENCES}

[1] L. Lapierre, D. Soetanto, and A. Pascoal, "Nonlinear path following with applications to the control of autonomous underwater vehicles," in Decision and Control, 2003. Proceedings. 42nd IEEE Conference on, vol. 2. IEEE, 2003, pp. 1256-1261.

[2] T. I. Fossen, Guidance and control of ocean vehicles. John Wiley \& Sons Inc, 1994.

[3] D. A. Smallwood and L. L. Whitcomb, "Model-based dynamic positioning of underwater robotic vehicles: theory and experiment," IEEE Journal of Oceanic Engineering, vol. 29, no. 1, pp. 169-186, 2004.

[4] D. Maalouf, I. Tamanaja, E. Campos, A. Chemori, V. Creuze, J. Torres, and R. Lozano, "From pd to nonlinear adaptive depth-control of a tethered autonomous underwater vehicle," IFAC Proceedings Volumes, vol. 46, no. 2, pp. 743-748, 2013.

[5] P. Sarhadi, A. R. Noei, and A. Khosravi, "Model reference adaptive pid control with anti-windup compensator for an autonomous underwater vehicle," Robotics and Autonomous Systems, vol. 83, pp. 87-93, 2016.

[6] R. Kelly and R. Carelli, "A class of nonlinear pd-type controllers for robot manipulators," Journal of Field Robotics, vol. 13, no. 12, pp. 793-802, 1996.

[7] E. Campos, A. Chemori, V. Creuze, J. Torres, and R. Lozano, "Saturation based nonlinear depth and yaw control of underwater vehicles with stability analysis and real-time experiments," Mechatronics, vol. 45, pp. 49-59, 2017.

[8] M. H. Khodayari and S. Balochian, "Modeling and control of autonomous underwater vehicle (auv) in heading and depth attitude via self-adaptive fuzzy pid controller," Journal of Marine Science and Technology, vol. 20, no. 3, pp. 559-578, 2015.

[9] R. Cui, C. Yang, Y. Li, and S. Sharma, "Adaptive neural network control of auvs with control input nonlinearities using reinforcement learning," IEEE Transactions on Systems, Man, and Cybernetics: Systems, vol. 47, no. 6, pp. 1019-1029, 2017.

[10] J.-H. Li and P.-M. Lee, "Design of an adaptive nonlinear controller for depth control of an autonomous underwater vehicle," Ocean engineering, vol. 32, no. 17-18, pp. 2165-2181, 2005.

[11] C. Yu, X. Xiang, Q. Zhang, and G. Xu, "Adaptive fuzzy trajectory tracking control of an under-actuated autonomous underwater vehicle subject to actuator saturation," International Journal of Fuzzy Systems, vol. 20, no. 1, pp. 269-279, 2018.

[12] N. Wang, S.-F. Su, J. Yin, Z. Zheng, and M. J. Er, "Global asymptotic model-free trajectory-independent tracking control of an uncertain marine vehicle: an adaptive universe-based fuzzy control approach," IEEE Transactions on Fuzzy Systems, vol. 26, no. 3, pp. 1613-1625, 2018.

[13] Y. Wang, L. Gu, M. Gao, and K. Zhu, "Multivariable output feedback adaptive terminal sliding mode control for underwater vehicles," Asian Journal of Control, vol. 18, no. 1, pp. 247-265, 2016.

[14] L. G. García-Valdovinos, T. Salgado-Jiménez, M. Bandala-Sánchez, L. Nava-Balanzar, R. Hernández-Alvarado, and J. A. Cruz-Ledesma, "Modelling, design and robust control of a remotely operated underwater vehicle," International Journal of Advanced Robotic Systems, vol. 11, no. 1, p. 1, 2014.

[15] H. Joe, M. Kim, and S.-c. Yu, "Second-order sliding-mode controller for autonomous underwater vehicle in the presence of unknown disturbances," Nonlinear Dynamics, vol. 78, no. 1, pp. 183-196, 2014.

[16] T. Salgado-Jiménez, L. G. García-Valdovinos, and G. Delgado-Ramírez, "Control of rovs using a model-free 2nd-order sliding mode approach," in Sliding Mode Control. InTech, 2011.

[17] J. Guerrero, J. Torres, E. Antonio, and E. Campos, "Autonomous underwater vehicle robust path tracking: Generalized super-twisting algorithm and block backstepping controllers," Journal of Control Engineering and Applied Informatics, vol. 20, no. 2, pp. 51-63, 2018. 
[18] I. D. Landau, R. Lozano, M. M'Saad, and A. Karimi, Adaptive control: algorithms, analysis and applications. Springer Science \& Business Media, 2011.

[19] D. Maalouf, A. Chemori, and V. Creuze, "L1 adaptive depth and pitch control of an underwater vehicle with real-time experiments," Ocean Engineering, vol. 98, pp. 66-77, 2015.

[20] J. Kim, H. Joe, S.-c. Yu, J. S. Lee, and M. Kim, "Time-delay controller design for position control of autonomous underwater vehicle under disturbances," IEEE Transactions on Industrial Electronics, vol. 63, no. 2, pp. 1052-1061, 2016.

[21] Z. H. Ismail and V. W. Putranti, "Second order sliding mode control scheme for an autonomous underwater vehicle with dynamic region concept," Mathematical Problems in Engineering, vol. 2015, 2015.

[22] J. Guerrero, J. Torres, V. Creuze, and A. Chemori, "Trajectory tracking for autonomous underwater vehicle: An adaptive approach,” Ocean Engineering, vol. 172, pp. 511-522, 2019.

[23] L. Qiao and W. Zhang, "Adaptive second-order fast nonsingular terminal sliding mode tracking control for fully actuated autonomous underwater vehicles," IEEE Journal of Oceanic Engineering, no. 99, pp. 1-23, 2018.

[24] — " "Double-loop integral terminal sliding mode tracking control for uuvs with adaptive dynamic compensation of uncertainties and disturbances," IEEE Journal of Oceanic Engineering, no. 99, pp. 1-25, 2018.

[25] P. Londhe, D. D. Dhadekar, B. Patre, and L. Waghmare, "Uncertainty and disturbance estimator based sliding mode control of an autonomous underwater vehicle," International Journal of Dynamics and Control, vol. 5, no. 4, pp. 1122-1138, 2017.

[26] Y. Qu, B. Xiao, Z. Fu, and D. Yuan, "Trajectory exponential tracking control of unmanned surface ships with external disturbance and system uncertainties," ISA Transactions, 2018.

[27] R. Cui, L. Chen, C. Yang, and M. Chen, "Extended state observer-based integral sliding mode control for an underwater robot with unknown disturbances and uncertain nonlinearities," IEEE Transactions on Industrial Electronics, vol. 64, no. 8, pp. 6785-6795, 2017.

[28] J. A. Moreno, "A linear framework for the robust stability analysis of a generalized super-twisting algorithm," in Electrical Engineering, Computing Science and Automatic Control, CCE, 2009 6th International Conference on. IEEE, 2009, pp. $1-6$.

[29] T. I. Fossen, Marine control systems: guidance, navigation and control of ships, rigs and underwater vehicles, 2002.

[30] T. T. J. Prestero, "Verification of a six-degree of freedom simulation model for the remus autonomous underwater vehicle," Ph.D. dissertation, Massachusetts institute of technology, 2001.

[31] J. C. Kinsey, R. M. Eustice, and L. L. Whitcomb, "A survey of underwater vehicle navigation: Recent advances and new challenges," in IFAC Conference of Manoeuvering and Control of Marine Craft, vol. 88, 2006.

[32] S. of Naval Architects, M. E. U. Technical, and R. C. H. Subcommittee, Nomenclature for Treating the Motion of a Submerged Body Through a Fluid: Report of the American Towing Tank Conference, ser. Technical and research bulletin. Society of Naval Architects and Marine Engineers, 1950. [Online]. Available: https://books.google.com.mx/books?id=sZ_bOwAACAAJ

[33] S. Soylu, B. J. Buckham, and R. P. Podhorodeski, "A chattering-free sliding-mode controller for underwater vehicles with fault-tolerant infinity-norm thrust allocation," Ocean Engineering, vol. 35, no. 16, pp. 1647-1659, 2008.

[34] J. A. Moreno, "Lyapunov approach for analysis and design of second order sliding mode algorithms," in Sliding Modes after the first decade of the 21st Century. Springer, 2011, pp. 113-149.

[35] J. Han, "A class of extended state observers for uncertain systems," Control and decision, vol. 10, no. 1, pp. 85-88, 1995.

[36] — "Auto-disturbance rejection control and its applications," Control and decision, vol. 13, no. 1, pp. 19-23, 1998.

[37] Y. Xia, Z. Zhu, M. Fu, and S. Wang, "Attitude tracking of rigid spacecraft with bounded disturbances," IEEE Transactions on Industrial Electronics, vol. 58, no. 2, pp. 647-659, 2011. 
Jesús Guerrero received his B.S. degree in Electronics and Communication Engineering from the University of Guanajuato, Mexico in 2012, and the M.Sc. degree in Automatic Control from the University of Queretaro, Mexico in 2014. He is currently pursuing Ph.D. degree in Automatic Control with the Center for Research and Advanced Studies of the National Polytechnic Institute, Mexico. His research interests include nonlinear, adaptive and time-delay control and their applications in underactuated systems, ground, aerial, and underwater vehicles.

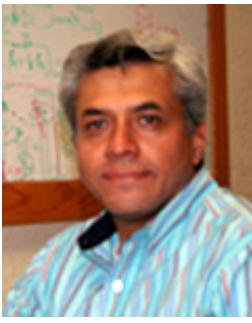

Jorge Torres was born in Mexico City, on May 13, 1960. He received the B.S. degree in Electronic Engineering from the National Polytechnic Institute (IPN) of Mexico in 1982, the M.S. degree in Electrical Engineering from CINVESTAV-IPN, Mexico in 1985, and the Ph.D. degree in Automatic Control from LAG, INPG, France, in 1990. He joined the Department of Electrical Engineering at the CINVESTAV, Mexico, in 1990. He spent a sabbatical year, from September 1997 to August 1998, at the Institute of Research in Communications and Cybernetics, IRCCYN-Nantes, France. Then, he served has the head of the Department of Automatic Control since its creation in September 1999 until January 2003, when he was called to serve as Secretary of Planning as a member of the Direction team of CINVESTAV, until March 2004. He was leading, from the Mexican side, the French Mexican Laboratory on Applied Automation (LAFMAA) of CNRS from January 2002 to January 2006. He was nominated as Deputy Director of the UMI 3175 LAFMIA at CINVESTAV Mexico, which is a joint research laboratory founded by CNRS, CINVESTAV and CONACYT for the period 20082012. His research interest lies in the structural approach of linear systems, stability of multivariate polynomials, and control of bioprocess for waste water treatment and control of mini-submarines

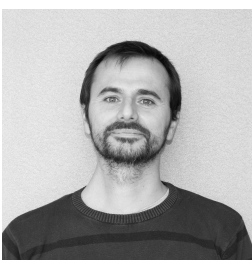

Vincent Creuze received his Ph.D. degree in 2002 in robotics from the University Montpellier 2, France. He is currently an associate professor at the University Montpellier 2, attached to the Robotics Department of the LIRMM (Montpellier Laboratory of Computer Science, Robotics, and Microelectronics). His research interests include design, modelling, and control of underwater robots, as well as underwater computer vision. 
Ahmed Chemori received his M.Sc. and Ph.D. degrees respectively in 2001 and 2005, both in automatic control from the Grenoble Institute of Technology. He has been a Post-doctoral fellow with the Automatic control laboratory of Grenoble in 2006. He is currently a tenured research scientist in Automatic control and Robotics at the Montpellier Laboratory of Informatics, Robotics, and Microelectronics. His research interests include nonlinear, adaptive and predictive control and their applications in humanoid robotics, underactuated systems, parallel robots, and underwater vehicles. 